Research Articl

\title{
Research on the Combination Method of Temporal Conflict Evidence Based on Transition Matrix
}

\author{
Bin Wu and Xiao Yi iD \\ Naval Aviation University, Yantai 264001, China \\ Correspondence should be addressed to Xiao Yi; yxgx_gxyx@163.com
}

Received 5 December 2019; Accepted 11 March 2020; Published 2 April 2020

Academic Editor: Josefa Mula

Copyright $(2020$ Bin Wu and Xiao Yi. This is an open access article distributed under the Creative Commons Attribution License, which permits unrestricted use, distribution, and reproduction in any medium, provided the original work is properly cited.

Conflict evidence combination is an important research topic in evidence theory. In this paper, two kinds of transition matrices are constructed based on the Markov model; one is the unordered transition matrix, which satisfies the commutative law, and the other is the temporal transition matrix, which does not satisfy the commutative law, but it can handle the combination of temporal evidence well. Then, a temporal conflict evidence combination model is proposed based on these two transition matrices. First, the transition probability at the first $n$ time is calculated through the model of unordered transition probability, and then, the transition matrix from the $N+1$ time is used to solve the combination problem of temporal conflict evidence. The effectiveness of the transition matrix in the research of conflict evidence combination method is proved by the example analysis.

\section{Introduction}

Since the evidence theory was put forward by Dempster [1] in 1976, many experts and scholars have made in-depth research on the theory and applied it to the fields of uncertain reasoning, multisource information fusion, pattern recognition, and so on, and the evidence theory achieved good performance. However, in the application, it is found that abnormal information (i.e., conflict evidence) is always output due to environmental disturbance or human interference. How to deal with these conflict evidence groups is related to the normal operation of the whole system, which has great significance. At present, the methods for resolving conflict evidence are mainly divided into two categories: one is to revise the evidence combination formula, which can be divided into three aspects: allocating conflict coefficients [2-5], changing combination rules [6], and expanding the recognition framework [7]. For example, Jiang proposes a new correlation coefficient considering the nonintersection between focus elements and the difference between focus elements for the problems of unstable or insensitive quantization confidence of existing correlation coefficients in the aspect of allocating conflict coefficients; in the aspect of changing combination rules, the Murphy additive combination rule is proposed to solve the problem existed in the multiplicative rule of DS evidence theory; in the aspect of the expanding recognition framework, DSmT is developed, which changes the original recognition framework and extends it to the generalized power set. The other is to revise the original data, which can be divided into two aspects: one is to describe the uncertainty of evidence by distance, and the other is based on information entropy.

There are two methods to measure the uncertainty of evidence by distance: the first is to measure the distance from point to point. Jousselme proposed the Jousselme [8] distance based on evidence. This distance function takes into account the potential of elements and is currently the most widely used evidence distance function. This solves the problem about the intersection of focal elements in the literature [9]; a similarity measurement [10] was proposed, which extended Jousselme distance. There are also similar measures of similarity proposed in reference [11]. The second is interval distance; in the interval distance, the relationship between focal belief and plausibility is fully considered, and the uncertain information is represented by the interval to calculate the distance between evidence groups

more accurately. For example, TD-IND interval distance is proposed in reference [12]. Generally, the purpose 
of these methods is to measure the uncertainty of evidence through distance and then correct the original data accordingly.

With the information entropy approach, the uncertainty of the evidence is characterized by entropy [13-16], which is based on the information carried by the evidence itself. Deng [17] proposed a new measure based on the entropy of basic probability distribution uncertainty, which is an extension of Shannon entropy. When BPA defines probability measure, Deng entropy has the same value as Shannon entropy. Yager [18] proposed Shannontype and Gini-type expressions of interval entropy. Zhou et al. [19] proposed an improved information entropy under the Dempster-Shafer framework, considering more information available in the body of evidence. The essence of these methods is that the evidence itself carries information, so its uncertainty can be characterized by entropy.

Through the above literature, we find that the research on conflict evidence is almost based on uncertain information, which is the revision of conflict evidence at a certain time or under the general concept of time. In this case, all the source data are obtained at one time, and there is little research on whether the fusion order affects the fusion results. However, it is impossible to complete the acquisition and fusion of all source information at one time in a complex system, so it has practical significance to study the influence of fusion sequence on fusion results.

The fusion order is largely reflected in the time series, so this paper studies the evidence combination of temporal conflicts. Hong and Lynch [20]. proposed a centralized/distributed recursive algorithm for spatiotemporal information integration, but they did not give a specific algorithm for temporal evidence fusion. Fan et al. [21]. obtained the reliability factor of evidence source through the evaluation method of intuitionistic fuzzy multicriteria and then used the factor to complete the timing evidence combination method with DS combination rules. Similar algorithms can be seen in references [22-27]. Li et al. [28]. constructed the spatiotemporal adjacent subsequences as information sources by splitting the image sequence and completed the fusion through the DS theory. The algorithm proposed by Huerta [29] provides time information for fusion through the location and speed of the target on the tracker. Dan et al. [30]. proposed a heuristic DS method to determine the relationship between local track and fusion tracks. Park and Chang [31] and others used the DS theory to express and combine the estimated probabilities of three different statistical models and eliminated the probabilities of unknown states through the orthogonal sum of probabilities. The above literature shows that most of the fusion of temporal evidence is based on application. There is no unified method to reflect the influence of time factors on the fusion of temporal evidence fully. Therefore, this paper focuses on the characteristics of temporal evidence to build a unified temporal evidence fusion framework.

\section{Theoretical Overview}

2.1. Evidence Theory. Evidence theory [1] is a kind of uncertain reasoning method which is oriented to the power set of the basic hypothesis set in the recognition framework, and it is suitable for different levels of sensor measurement. Let $\Theta$ be the recognition framework, and the basic belief assignment $m$ is a mapping from the set $2^{\Theta}$ to $\left[\begin{array}{ll}0 & 1\end{array}\right]$, and $A$ represents any subset of the recognition framework $\Theta$, which is recorded as $A \subseteq \Theta$, and satisfies

$$
\left\{\begin{array}{l}
m(\varnothing)=0, \\
\sum_{A \subset \Theta} m(A)=1,
\end{array}\right.
$$

where $m(A)$ is the basic belief function of the event $A$, which represents the trust degree of event $A$.

2.2. Belief Function and Plausibility Function. Belief function and plausibility function are defined as

$$
\begin{gathered}
\operatorname{Bel}(A)=\sum_{B \subseteq A} m(B), \\
P l(A)=\sum_{B \cap A \neq \varnothing} m(B),
\end{gathered}
$$

where the confidence interval $[\operatorname{Bel}(A) \operatorname{Pl}(A)]$ represents the uncertainty of event $A$.

The evidence combination rules of DS evidence theory are as follows:

$$
m(A)= \begin{cases}\frac{\sum_{A_{i} \cap B_{j}=A} m_{1}\left(A_{i}\right) m_{2}\left(B_{j}\right)}{1-K}, & A \neq \varnothing, \\ 0, & A=\varnothing,\end{cases}
$$

where $K=\sum_{A_{i} \cap B_{j}=\varnothing} m_{1}\left(A_{i}\right) m_{2}\left(B_{j}\right)$. In classic evidence theory, the coefficient $K$ represents the degree of conflict between evidence.

2.3. Model of Markov chain. Markov chain is a set of discrete random variables with Markov properties. Specifically, for $t_{1}<t_{2}<\cdots<t_{n}, P\left[x\left(t_{n}\right)=x_{n} \mid x\left(t_{n-1}\right), \ldots, x\left(t_{1}\right)\right]=P\left[x\left(t_{n}\right)=\right.$ $\left.x_{n} \mid x\left(t_{n-1}\right)\right]$. Its time states are discrete Markov processes.

Definition 1 Transition Probability (see $[32,33]$ ). In the discrete Markov chain $\left\{x_{n}\right\}$, its state can be expressed as $s_{1}, s_{2}, \ldots, s_{i}, \ldots, x_{n}=x\left(t_{n}\right)$ in the state of the system at $t=t_{n}$, when $0 \leq m \leq n$ and sequence $x_{m} \longrightarrow x_{m+1} \longrightarrow x_{n}$, which represents the evolution of the system. $p_{i}(m)=P\left\{x_{m}=s_{i}\right\}$ represents the probability of state $s_{i}$ at $t=t_{m} . \quad p_{i j}(m, n)=P\left\{x_{n}=s_{j} \mid x_{m}=s_{i}\right\} \quad$ represents the probability that the system will become state $s_{j}$ at $t_{n}$ when the system is in state $s_{i}$ at $t_{m} . p_{i j}(m, n)$ is the transition probability.

Definition 2 Transition Matrix (see [32, 33]). Matrix $P(m, n)$ is composed of the transition probability $p_{i j}(m, n)$ : 


$$
P(m, n)=\left[\begin{array}{ccccc}
p_{11}(m, n) & p_{12}(m, n) & \cdots & p_{1 j}(m, n) & \cdots \\
p_{21}(m, n) & p_{22}(m, n) & \cdots & \cdots & \cdots \\
\vdots & \vdots & \ddots & \vdots & \vdots \\
p_{i 1}(m, n) & \cdots & \cdots & p_{i j}(m, n) & \cdots \\
\cdots & \cdots & \cdots & \cdots & \cdots
\end{array}\right]
$$

where $\quad p_{i j}(m, n)>0 \quad$ and $\quad \sum_{j} p_{i j}(m, n)=\sum_{j} P\left\{x_{n}=s_{j}\right.$ $\left.\mid x_{m}=s_{i}\right\}=1$. Specifically, $P(m, n)$ is called the one-step transition probability matrix when $n=m+1$. There are other definitions and properties of Markov chain, such as homogeneous chain and c-k equation, which can be found in $[32,33]$, and will not be discussed in this paper.

\section{Transition Matrix Based on the Markov Chain Model}

3.1. Transition Matrix of Evidence. A random process was described by the Markov model. This stochastic process meets an important property, which is that the next state only depends on the current state and has nothing to do with all past states. The state at the next time is different from the past. The states are conditionally independent, so this random process has Markov properties. The second section of the paper has introduced the related theory of Markov chain. In this paper, Markov's idea will be used to propose the transition probability matrix of evidence belief.

Evidence describes the degree of trust for the source in evidence theory. Because the source is inevitably interfered by external factors in the process of obtaining information, the received information has certain randomness. However, the information obtained by the source at different time points is independent of each other. Suppose the information obtained by the source at different time points is recorded as

$$
\begin{aligned}
& m_{1}=\{A: 0.6, B: 0.2, A B: 0.1, C: 0, A C: 0.05, B C: 0.05\} \\
& m_{2}=\{A: 0.5, B: 0.1, A B: 0.15, C: 0.05, A C: 0.1, B C: 0.1\} \\
& m_{3}=\{A: 0.7, B: 0, A B: 0.1, C: 0.05, A C: 0.15, B C: 0\}
\end{aligned}
$$

Here, the belief value obtained by the source at three time points is given. Considering the one-step transition probability between the belief, that is, $m_{i} \longrightarrow m_{j}, i \neq j$, recorded as $p_{A \& B}$, which represents the transition probability between $A$ and $B$ focal elements between evidence, calculates the transition probability between every two focal elements, and obtains a transition probability matrix,

$$
P=\left[\begin{array}{cccc}
p_{A \& A} & p_{A \& B} & \cdots & p_{A \& B C} \\
p_{B \& A} & p_{B \& B} & \cdots & p_{B \& B C} \\
\vdots & \vdots & \ddots & \vdots \\
p_{B C \& A} & p_{B C \& B} & \cdots & p_{B C \& B C}
\end{array}\right]
$$

Therefore, in order to obtain the transition matrix, we must first determine the transition probability between two focal elements in the evidence, that is, the problem of obtaining the probability $p_{A \& B}$.

In evidence theory, there are many combination rules, and the most classic one is the Dempster combination rule. In the Dempster combination rule, the degree of correlation between evidence is expressed by the product of two focal elements. Because of this, this paper proposes the general transition probability between focal elements:

$$
p_{A \& B}^{\prime}=\frac{2}{n(n-1)} \sum_{i \neq j} m_{i}(A) m_{j}(B),
$$

where the product of the two beliefs is used directly to represent the transition probability between the two focal elements. But the evidence vector describes the belief, and the belief is different from the probability in probability theory. There is an intersection between each focal element in the reliability, and the potential of each focal element is different. The idea of using the potential of the focal element to weight the belief has been applied in many formulas, such as in the Jousselme evidence distance:

$$
d_{J}\left(m_{1}, m_{2}\right)=\sqrt{\frac{1}{2}\left(m_{1}-m_{2}\right)^{T} D_{J}\left(m_{1}-m_{2}\right)},
$$

where $D_{J}$ in the formula uses the potential of the focal element to modify the belief of the evidence and $D_{J}=\left[\left|A_{i} \cap A_{j}\right| /\left|A_{i} \cup A_{j}\right|\right]$.

In this paper, the coefficient $\left|A_{i} \cap A_{j}\right| /\left|A_{i} \cup A_{j}\right|$ is introduced into the evidence transition probability matrix to obtain the transition probability between the common focal elements:

$$
p_{A \& B}=\frac{2}{n(n-1)} \sum_{i \neq j} \frac{|A \cap B|}{|A \cup B|} m_{i}(A) m_{j}(B) .
$$

Equation (9) gives the general focal element probability conversion, but considering the actual problem, the evidence given by the source is sequential, and the general focal element transition probability is calculated on any two sets of evidence, assuming the evidence has $n$ group, and this requires calculating the transition probability between $n(n-$ 1)/2 groups of evidence. For example, the three pieces of evidence given above are taken as an example. Generally, the transition probability needs to be solved between groups $m_{1} \& m_{2}, m_{2} \& m_{3}$, and $m_{1} \& m_{3}$ :

$$
\begin{aligned}
P_{A \& A} & =\frac{2}{n(n-1)} \sum_{i \neq j} m_{i}(A) m_{j}(A) \\
& =\frac{1}{3}\left(m_{1}(A) m_{2}(A)+m_{1}(A) m_{3}(A)+m_{3}(A) m_{2}(A)\right) \\
& =\frac{1}{3}(0.3+0.35+0.42) \\
& \approx 0.357 .
\end{aligned}
$$


There are two problems. One is that when the number of evidence is large, the calculation amount of evidence transfer probability is very large, which increases exponentially, and the other is that it cannot deal with the combination of sequential evidence.

Therefore, for the combination of sequential evidence, this paper improves the general evidence transition matrix formula to obtain the sequential evidence transition probability:

$$
p_{A \& B}^{t}=\frac{1}{n-1} \sum_{i=1}^{n-1} \frac{|A \cap B|}{|A \cup B|} m_{i}(A) m_{i+1}(B) .
$$

The sequential evidence transition probability considers the independence between the evidence and does not consider the transition probability between any two groups, but only the transition probability between adjacent moments of evidence. The above three pieces of evidence are shown as follows:

$$
\begin{aligned}
p_{A \& A}^{t} & =\frac{1}{n-1} \sum_{i=1}^{n-1} m_{i}(A) m_{j}(A) \\
& =\frac{1}{2}\left(m_{1}(A) m_{2}(A)+m_{3}(A) m_{2}(A)\right) \\
& =\frac{1}{2}(0.3+0.42) \\
& =0.360 .
\end{aligned}
$$

The following paper analyzes the exchange law properties of the two proposed evidence transition matrices.

By analyzing the general transfer probability formula $(2 / n(n-1)) \sum_{i \neq j} m_{i}(A) m_{j}(B)$, it can be seen that since the formula solves the transition probability between every two pieces of evidence, the formula satisfies the exchange law, while the sequential evidence transition probability formula $(1 /(n-1)) \sum_{i=1}^{n-1}(|A \cap B| /|A \cup B|) m_{i}(A) m_{i+1}(B)$ only solves the transfer probability between two adjacent pieces of evidence, so the formula does not satisfy the exchange law. For example, if the three pieces of evidence $m_{1}, m_{2}$, and $m_{3}$ above are received in the order $m_{1}, m_{3}$, and $m_{2}$, the transition probability of the focal element $A \longrightarrow A$ is

$$
\begin{aligned}
p_{A \& A}^{t} & =\frac{1}{n-1} \sum_{i=1}^{n-1} m_{i}(A) m_{j}(A), \\
& =\frac{1}{2}\left(m_{1}(A) m_{3}(A)+m_{3}(A) m_{2}(A)\right) \\
& =\frac{1}{2}(0.35+0.42) \\
& =0.385 \neq 0.360,
\end{aligned}
$$

so the sequential evidence transition probability does not satisfy the exchange law.

Then, the computation of the two transition probability matrices is analyzed. The general transition probability matrix has been analyzed above, and the computation is $(n(n-1) / 2) m$. The sequential transition matrix only considers the transition probability between two adjacent pieces of evidence. The computation is $(n-1) m$, where $m=\left(2^{|\Theta|}\right)^{2}$ is the square of the dimension of the transition probability matrix.

3.2. Effectiveness Analysis of Transition Matrix. First, the validity of the general evidence transition probability matrix is analyzed. Since the computation of general evidence transition probability increases exponentially with the number of evidence, this article only considers 6 pieces of evidence. The values of the 6 pieces of evidence obtained by the source are shown in Table 1.

According to Table 1, calculating through the general evidence transition probability formula, the transition probability matrix is as shown in Table 2.

Then, the 6 pieces of evidence are transformed through the transition matrix (Table 2) to obtain a new group of evidence, which is shown in Table 3.

By comparing the evidence groups before and after the transfer, it can be found that the similarity before and after the transfer is high, and the evidence changes after the transfer are relatively stable. In order to qualitatively analyze the change degree before and after the transfer of evidence, the distance between each group of evidence and the original evidence group is calculated, respectively, and the results are as shown in Figure 1.

In Figure 1, the horizontal axis represents the transformed evidence. There are 6 polylines, which represent the 6 pieces of evidence before the transformation. The vertical axis represents the distance between the two pieces of evidence. The distance used here is the Jousselme evidence distance widely used in evidence theory. Two conclusions are obtained by analyzing the data in the table. One is that the distance between the converted evidence and the original evidence group is relatively small; that is, the converted evidence group is relatively similar to the original evidence group. Second, it is found that the trend of each line is similar; that is, the distance between each piece of evidence after conversion is relatively similar, and the degree of similarity between the two pieces of converted evidence is higher.

For the analysis of the validity of temporal evidence, the article will give a more detailed analysis in Section 5 .

\section{Conflict Evidence Combination Model Based on the Transition Probability Matrix}

4.1. Conflict Evidence Combination Model. The solution method of the evidence transition probability matrix is given above, and the validity of the transition probability is analyzed. However, it can be seen from the calculation examples that there is no conflict between the evidence. However, in actual engineering applications, there may be conflicts between different sources or even the same source at different time points. This conflict may come from hardware disturbances or external interference. How to combine conflict evidence is an important research topic of evidence theory. 
TABLE 1: Values of 6 pieces of evidence.

\begin{tabular}{cccccccc}
\hline & $A$ & $B$ & $C$ & $A B$ & $A C$ & $B C$ & $A B C$ \\
\hline 1 & 0.55 & 0.1 & 0.1 & 0.1 & 0.1 & 0.05 & 0 \\
2 & 0.6 & 0.1 & 0.05 & 0.1 & 0.1 & 0.05 & 0 \\
3 & 0.5 & 0.1 & 0.2 & 0.1 & 0.05 & 0.05 & 0 \\
4 & 0.65 & 0 & 0.1 & 0.1 & 0.1 & 0.05 & 0 \\
5 & 0.7 & 0.05 & 0.05 & 0.1 & 0.1 & 0 & 0 \\
6 & 0.5 & 0.05 & 0 & 0.25 & 0.2 & 0 & 0 \\
\hline
\end{tabular}

TABLE 2: Specific values of the transition matrix.

\begin{tabular}{lccccccc}
\hline & $A$ & $B$ & $C$ & $A B$ & $A C$ & $B C$ & $A B C$ \\
\hline$A$ & 0.8375 & 0 & 0.0531 & 0 & 0.1094 & 0 & 0 \\
$B$ & 0 & 0.4647 & 0.4015 & 0 & 0 & 0.1338 & 0 \\
$A B$ & 0.7103 & 0.0555 & 0.1181 & 0 & 0.1008 & 0.0153 & 0 \\
$C$ & 0 & 0 & 0 & 0.6303 & 0.3193 & 0.0504 & 0 \\
$A C$ & 0.5849 & 0 & 0.0431 & 0.1494 & 0.2091 & 0.0135 & 0 \\
$B C$ & 0 & 0.1409 & 0.1275 & 0.4027 & 0.2282 & 0.1007 & 0 \\
$A B C$ & 0 & 0 & 0 & 0 & 0 & 0 & 0 \\
\hline
\end{tabular}

TABLE 3: New group of evidence.

\begin{tabular}{cccccccc}
\hline & $A$ & $B$ & $C$ & $A B$ & $A C$ & $B C$ & $A B C$ \\
\hline 1 & 0.5901 & 0.0591 & 0.0918 & 0.0981 & 0.1345 & 0.0263 & 0 \\
2 & 0.5965 & 0.0563 & 0.0886 & 0.0981 & 0.1349 & 0.0256 & 0 \\
3 & 0.5901 & 0.0646 & 0.0988 & 0.0906 & 0.1287 & 0.0272 & 0 \\
4 & 0.6739 & 0.0126 & 0.0570 & 0.0981 & 0.1454 & 0.0130 & 0 \\
5 & 0.6803 & 0.0260 & 0.0674 & 0.0780 & 0.1345 & 0.0139 & 0 \\
6 & 0.5357 & 0.0232 & 0.0552 & 0.1875 & 0.1763 & 0.0220 & 0 \\
\hline
\end{tabular}

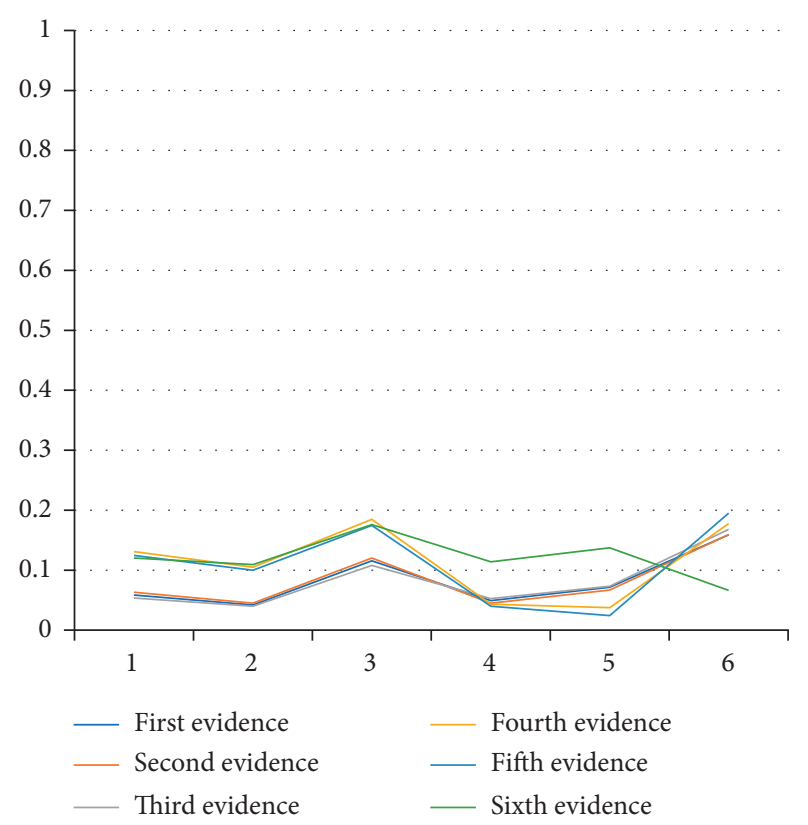

FIgURE 1: Comparison chart of evidence results.

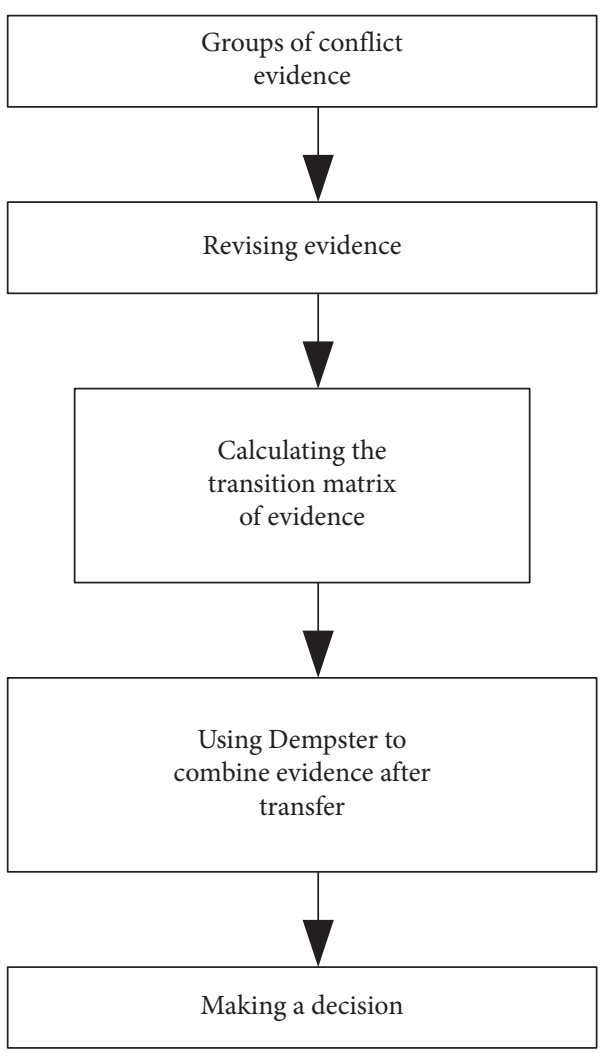

FIGURE 2: Flow chart of the conflict evidence combination model.

This section will introduce how to use the evidence transition probability matrix to effectively fuse conflict evidence. The conflict evidence combination model is given in Figure 2.

In Figure 2, the first step is to modify the conflicting evidence values, then calculate the transition probability matrix of the modified evidence group, and finally use the Dempster combination rule to combine the transferred evidence groups to obtain the combined evidence for decision-making.

There are many ways to modify evidence; the common one is to put forward a new formula of evidence distance or a measure index that can measure the difference between evidence, calculate the support degree between evidence, use the support degree of evidence to represent the credibility of evidence, and then modify the evidence.

The method of conflict evidence correction based on interval distance is given in the next section.

\subsection{Correction of Conflict Evidence Based on IntervalDistance.} In this section, the interval evidence distance is proposed and the interval evidence distance is used to modify the evidence. Assuming that the recognition framework is $\Theta=$ $\left\{\theta_{1}, \theta_{2}, \theta_{3}, \ldots, \theta_{n}\right\}$ and $m_{i}(i=1,2,3, \ldots, n)$ are the pieces of evidence under the recognition framework, and the basic 
trust belief function is $m_{j}\left(A_{i}\right)$, which represents the $i$ th focal element of the piece of evidence $j$, the uncertainty interval $A_{i}$ is expressed as $\left[\operatorname{Bel}_{j}\left(A_{i}\right) \quad P l_{j}\left(A_{i}\right)\right]$.
The distance of the evidence uncertainty interval is $d\left(m_{j}\left(A_{i}\right), m_{k}\left(A_{i}\right)\right)$ :

$$
d\left(m_{j}\left(A_{i}\right), m_{k}\left(A_{i}\right)\right)=\frac{\sqrt{2}}{2} \cdot \sqrt{\left(\operatorname{Bel}_{j}\left(A_{i}\right)-\operatorname{Bel}_{k}\left(A_{i}\right)^{2}+\left(P l_{j}\left(A_{i}\right)-P l_{k}\left(A_{i}\right)\right)\right)^{2}} .
$$

This represents the interval evidence distance between the $j$ th and $m$ th evidence, and the total distance between the two pieces of evidence can be expressed as

$$
d_{j k}=\frac{1}{2^{|\Theta|}} \sum d\left(m_{j}, m_{k}\right)
$$

The interval evidence distance of each focal element is solved, and then, the mean value is taken to represent the distance between evidence groups.

Calculate the distance between every two pieces of evidence to get the distance matrix $D$ :

$$
D=\left[\begin{array}{cccc}
0 & d_{12} & \cdots & d_{1 n} \\
d_{21} & 0 & \cdots & d_{2 n} \\
& & \vdots & \\
d_{n 1} & d_{n 2} & \cdots & 0
\end{array}\right] .
$$

Sum the evidence distance $D$ and get the distance vector $d=\left[d_{1}, d_{2}, \ldots, d_{n}\right]$, where $d_{i}$ is the distance between the $i$ th evidence and the whole evidence group.

Then normalize the distance vector to get the normalized distance vector

$$
d^{\prime}=\left[d_{1}^{\prime}, d_{2}^{\prime}, \ldots, d_{n}^{\prime}\right]
$$

where $d_{i}^{\prime}=d_{i} / \operatorname{sum}(d)$.

Through the distance matrix, calculate the degree of support for each piece of evidence:

$$
s=\left[s_{1}, s_{2}, \ldots, s_{n}\right],
$$

where $s_{i}=1-d_{i}^{\prime}$ indicates the degree to which the $i$ thevidence is supported by the entire evidence group. Then, use the support vector $s$ to modify the evidence to get $m_{i}^{\prime}=s_{i} m_{i} i$.

In the following, we will analyze the correction of conflict evidence and give a combination of conflict evidence and analysis of the effectiveness of the model.

\subsection{Effectiveness Analysis of the Conflict Evidence Combi-} nation Model. According to the above model, the validity analysis of the combined model of conflict evidence is based on the evidence transition probability matrix. The values of 7 pieces of evidence received by the source are shown in Table 4.

By analyzing the data in Table 4 , we can see that there is a clear conflict between the 7th evidence and the first 6 pieces of evidence. The following model will be used to combine these 7 evidence groups.
The first step is to calculate the belief function and the plausibility function of the evidence, which is shown in Table 5.

The elements in Table 5 represent the interval distance $[\mathrm{Bel}, \mathrm{Pl}]$. The second step is to calculate the distance between every two pieces of evidence according to the above interval distance to obtain the distance matrix. The specific value of the distance is shown in Table 6.

Then, sum the matrices to get the distance vector:

$d=\left[\begin{array}{lllllll}2.2400 & 2.3900 & 2.4100 & 3.1600 & 3.5300 & 3.4400 & 12.8300\end{array}\right]$.

The normalized distance matrix is obtained by normalizing the distance vector:

$d^{\prime}=\left[\begin{array}{lllllll}0.0747 & 0.0797 & 0.0803 & 0.1053 & 0.1177 & 0.1147 & 0.4277\end{array}\right]$.

Then, according to the distance matrix, the support vector is calculated:

$s=\left[\begin{array}{lllllll}0.9253 & 0.9203 & 0.9197 & 0.8947 & 0.8823 & 0.8853 & 0.5723\end{array}\right]$.

Then, the support vector is used to modify the evidence, and a new group of evidence is obtained, which is shown in Table 7.

Then, according to Table 7 , the transition probability matrix of the modified evidence is obtained (seen from Table 8).

The transition probability matrix is normalized (seen from Table 9).

Using the transition probability matrix (Table 9) to transfer the original evidence, the new group of evidence after the transfer is obtained (seen from Table 10).

Finally, the Dempster rule is used to combine 7 pieces of evidence through Table 10, and the final evidence is

$m=\left[\begin{array}{lllllll}0.9969 & 0.0002 & 0.0000 & 0.0026 & 0.0002 & 0.0000 & 0.0000\end{array}\right]$.

From the final calculation results, the conflict evidence combination method based on the evidence transfer probability matrix can obtain a good fusion effect.

This section is a combination of disordered evidence groups. The following will consider how to combine ordered evidence groups and give a reasonable model of sequential conflict evidence combination. 
TABLE 4: Values of 7 pieces of evidence.

\begin{tabular}{|c|c|c|c|c|c|c|c|}
\hline & $A$ & $B$ & $C$ & $A B$ & $A C$ & $B C$ & $A B C$ \\
\hline 1 & 0.55 & 0.1 & 0.1 & 0.1 & 0.1 & 0.05 & 0 \\
\hline 2 & 0.6 & 0.1 & 0.05 & 0.1 & 0.1 & 0.05 & 0 \\
\hline 3 & 0.5 & 0.1 & 0.2 & 0.1 & 0.05 & 0.05 & 0 \\
\hline 4 & 0.65 & 0 & 0.1 & 0.1 & 0.1 & 0.05 & 0 \\
\hline 5 & 0.7 & 0.05 & 0.05 & 0.1 & 0.1 & 0 & 0 \\
\hline 6 & 0.5 & 0.05 & 0 & 0.25 & 0.2 & 0 & 0 \\
\hline 7 & 0 & 0.5 & 0.1 & 0.1 & 0.2 & 0.1 & 0 \\
\hline
\end{tabular}

TABle 5: Values of belief and plausibility.

\begin{tabular}{lccccccc}
\hline & 1 & 2 & 3 & 4 & 5 & 6 \\
\hline$A$ & $0.55,0.75$ & $0.60,0.75$ & $0.50,0.75$ & $0.65,0.85$ & $0.70,0.85$ & $0.50,0.70$ & $0,0.20$ \\
$B$ & $0.10,0.25$ & $0.10,0.20$ & $0.10,0.35$ & $0,0.15$ & $0.05,0.10$ & $0.05,0.05$ & $0.50,0.70$ \\
$A B$ & $0.75,0.90$ & $0.75,0.90$ & $0.80,0.90$ & $0.75,0.90$ & $0.80,0.90$ & $0.55,0.75$ & $0.60,0.80$ \\
$C$ & $0.10,0.25$ & $0.10,0.25$ & $0.10,0.20$ & $0.10,0.25$ & $0.10,0.20$ & $0.25,0.45$ & $0.10,0.30$ \\
$A C$ & $0.75,0.90$ & $0.80,0.90$ & $0.65,0.90$ & $0.85,1$. & $0.90,0.95$ & $0.95,0.95$ & $0.20,0.40$ \\
$B C$ & $0.25,0.45$ & $0.25,0.40$ & $0.25,0.50$ & $0.15,0.35$ & $0.15,0.30$ & $0.30,0.50$ & $0.70,0.90$ \\
\hline
\end{tabular}

TABLE 6: Specific value of the distance between evidence groups.

\begin{tabular}{cccccccc}
\hline & 1 & 2 & 3 & 4 & 5 & 6 & 7 \\
\hline 1 & 0 & 0.0100 & 0.0300 & 0.0800 & 0.1200 & 0.2200 & 1.7800 \\
2 & 0.0100 & 0 & 0.0700 & 0.0500 & 0.0700 & 0.2000 & 1.9900 \\
3 & 0.0300 & 0.0700 & 0 & 0.1700 & 0.2300 & 0.3600 & 1.5500 \\
4 & 0.0800 & 0.0500 & 0.1700 & 0 & 0.0200 & 0.2400 & 2.6000 \\
5 & 0.1200 & 0.0700 & 0.2300 & 0.0200 & 0 & 0.3000 & 2.7900 \\
6 & 0.2200 & 0.2000 & 0.3600 & 0.2400 & 0.3000 & 0 & 2.1200 \\
7 & 1.7800 & 1.9900 & 1.5500 & 2.6000 & 2.7900 & 2.1200 & 0 \\
\hline
\end{tabular}

TABle 7: New group of evidence.

\begin{tabular}{cccccccc}
\hline & $A$ & $B$ & $C$ & $A B$ & $A C$ & $B C$ & $A B C$ \\
\hline 1 & 0.5089 & 0.0925 & 0.0925 & 0.0925 & 0.0925 & 0.0463 & 0.0747 \\
2 & 0.5522 & 0.0920 & 0.0460 & 0.0920 & 0.0920 & 0.0460 & 0.0797 \\
3 & 0.4598 & 0.0920 & 0.1839 & 0.0920 & 0.0460 & 0.0460 & 0.0803 \\
4 & 0.5815 & 0 & 0.0895 & 0.0895 & 0.0895 & 0.0447 & 0.1053 \\
5 & 0.6176 & 0.0441 & 0.0441 & 0.0882 & 0.0882 & 0 & 0.1177 \\
6 & 0.4427 & 0.0443 & 0 & 0.2213 & 0.1771 & 0 & 0.1147 \\
7 & 0 & 0.2862 & 0.0572 & 0.0572 & 0.1145 & 0.0572 & 0.4277 \\
\hline
\end{tabular}

TABLE 8: Transition probability matrix of the modified evidence.

\begin{tabular}{lccccccc}
\hline & $A$ & $B$ & $C$ & $A B$ & $A C$ & $B C$ & $A B C$ \\
\hline$A$ & 0.2771 & 0 & 0.0179 & 0 & 0.0360 & 0 & 0.0225 \\
$B$ & 0 & 0.0035 & 0.0030 & 0 & 0 & 0.0010 & 0.0032 \\
$A B$ & 0.0303 & 0.0024 & 0.0051 & 0 & 0.0043 & 0.0007 & 0.0072 \\
$C$ & 0 & 0 & 0 & 0.0122 & 0.0062 & 0.0010 & 0.0039 \\
$A C$ & 0.0259 & 0 & 0.0019 & 0.0066 & 0.0092 & 0.0006 & 0.0061 \\
$B C$ & 0 & 0.0012 & 0.0011 & 0.0033 & 0.0019 & 0.0008 & 0.0032 \\
$A B C$ & 0.0176 & 0.0015 & 0.0032 & 0.0046 & 0.0068 & 0.0010 & 0.0090 \\
\hline
\end{tabular}

TABLE 9: Normalized transition probability matrix.

\begin{tabular}{lccccccc}
\hline & $A$ & $B$ & $C$ & $A B$ & $A C$ & $B C$ & $A B C$ \\
\hline$A$ & 0.7839 & 0 & 0.0506 & 0 & 0.1017 & 0 & 0.0638 \\
$B$ & 0 & 0.3237 & 0.2813 & 0 & 0 & 0.0940 & 0.3011 \\
$A B$ & 0.6074 & 0.0477 & 0.1024 & 0 & 0.0859 & 0.0134 & 0.1432 \\
$C$ & 0 & 0 & 0 & 0.5239 & 0.2653 & 0.0431 & 0.1677 \\
$A C$ & 0.5145 & 0 & 0.0386 & 0.1307 & 0.1827 & 0.0122 & 0.1212 \\
$B C$ & 0 & 0.1019 & 0.0929 & 0.2881 & 0.1631 & 0.0737 & 0.2803 \\
$A B C$ & 0.0176 & 0.0015 & 0.0032 & 0.0046 & 0.0068 & 0.0010 & 0.0090
\end{tabular}

TABLE 10: New group of evidence.

\begin{tabular}{cccccccc}
\hline & $A$ & $B$ & $C$ & $A B$ & $A C$ & $B C$ & $A B C$ \\
\hline 1 & 0.5040 & 0.0392 & 0.0694 & 0.0742 & 0.1092 & 0.0185 & 0.1139 \\
2 & 0.5095 & 0.0368 & 0.0666 & 0.0738 & 0.1094 & 0.0178 & 0.1097 \\
3 & 0.4972 & 0.0434 & 0.0743 & 0.0678 & 0.1034 & 0.0191 & 0.1180 \\
4 & 0.5581 & 0.0090 & 0.0465 & 0.0719 & 0.1149 & 0.0095 & 0.0892 \\
5 & 0.5584 & 0.0166 & 0.0520 & 0.0583 & 0.1069 & 0.0097 & 0.0855 \\
6 & 0.4402 & 0.0145 & 0.0421 & 0.1396 & 0.1369 & 0.0160 & 0.1012 \\
7 & 0.1012 & 0.1019 & 0.0974 & 0.0634 & 0.0533 & 0.0362 & 0.1377 \\
\hline
\end{tabular}

\section{Temporal Conflict Evidence Combination Model}

5.1. Construction of the Temporal Conflict Evidence Combination Model. First, the general transfer probability matrix is calculated by the method given in Section 4 for the first $n$ time points, and then, the combined evidence is calculated. Then, the subsequent combination of temporal conflict evidence is carried out based on this evidence. The model is given in Figure 3.

As shown in Figure 3, first, determine whether there is a conflict between the evidence entered at the next moment 


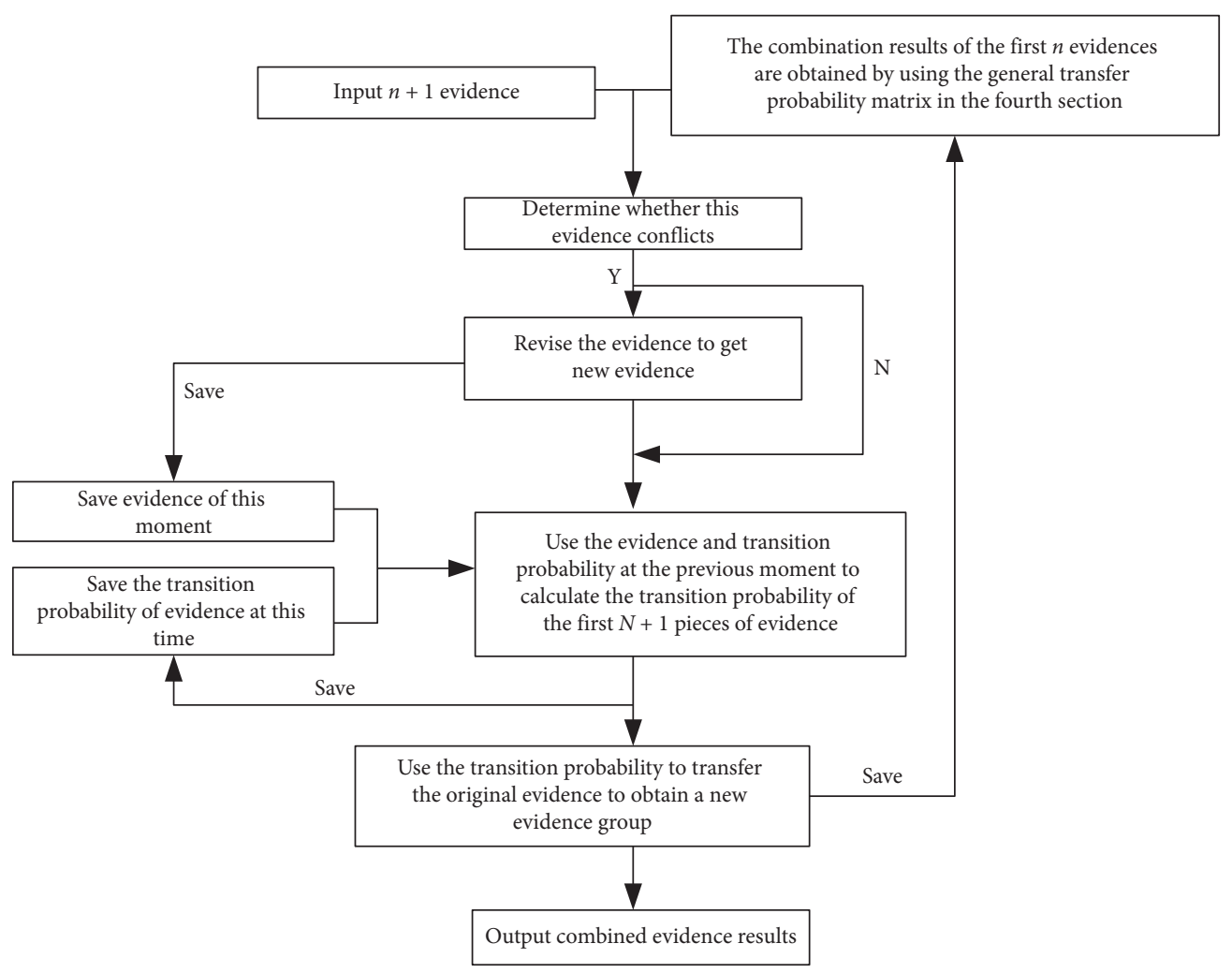

FIgURE 3: Flow chart of the temporal conflict evidence combination model.

and if there is a conflict, revise the evidence. Here, you can set a threshold to determine whether there is a conflict and then use the evidence from the previous moment to compare with the previous moment. The transition probability is calculated to obtain the transition probability at this moment, and the evidence at this moment and the transition probability at this moment are saved for calculation at the next moment.

After obtaining the transition probability, the original evidence group can be transferred through the transition probability to obtain a new evidence group, and then the combined result can be obtained through the combination formula.

The difficulty of the model lies in how to judge whether the evidence at this moment is in conflict and how to calculate the transition probability at the moment based on the evidence and transition probability at the previous moment. The specific calculation steps are given below, and the validity of the model is reasonably analyzed.

5.2. Validity Analysis of the Evidence Combination Model of Temporal Conflict. In order to facilitate the analysis of the problem, it is assumed that the seven pieces of evidence are those given in Section 4. In the previous section, the article has combined them with the general evidence transition probability matrix, although there is a conflict among the seven pieces of evidence. But it still can get a better combination effect.

According to the calculation results of Section 4, the status of this time is obtained, in which the evidence stored at this time is 6th evidence (because 7th is judged to be conflicting evidence, it will not be stored), that is,

$$
m_{n}=\left[\begin{array}{lllllll}
0.5 & 0.05 & 0 & 0.25 & 0.2 & 0 & 0
\end{array}\right] \text {. }
$$

The transition probability matrix at this moment is given in Table 11.

According to Table 11, the combined result at this moment is

$m_{N}=\left[\begin{array}{lllllll}0.9969 & 0.0002 & 0.0000 & 0.0026 & 0.0002 & 0.0000 & 0.0000\end{array}\right]$.

Supposing a new piece of evidence comes next time:

$$
m_{n+1}=\left[\begin{array}{lllllll}
0.6 & 0.05 & 0 & 0.15 & 0.2 & 0 & 0
\end{array}\right] \text {. }
$$

The first step is to calculate the distance between the two pieces of evidence $m_{N}$ and $m_{n+1}$. Here, the Jousselme distance is used as an example to obtain a distance of 0.2931 . The distance is small, which has no conflict, and no evidence correction is needed.

Save the evidence $m_{n+1}$ at this moment, and then, solve the evidence transition probability matrix at the next moment. Using $m_{n}$ and $m_{n+1}$ and the evidence transition matrix at the previous moment, calculate the transition probability matrix at this moment as shown in Table 12.

Then, according to Table 12, the evidence transition probability matrix at this moment is stored and used for solving the evidence transition probability matrix at the next moment. The next steps are similar to those in Section 4 . First, normalize the evidence transfer probability matrix and 
TABle 11: Transition probability matrix at some moment.

\begin{tabular}{lccccccc}
\hline & $A$ & $B$ & $C$ & $A B$ & $A C$ & $B C$ & $A B C$ \\
\hline$A$ & 0.2771 & 0 & 0.0179 & 0 & 0.0360 & 0 & 0.0225 \\
$B$ & 0 & 0.0035 & 0.0030 & 0 & 0 & 0.0010 & 0.0032 \\
$A B$ & 0.0303 & 0.0024 & 0.0051 & 0 & 0.0043 & 0.0007 & 0.0072 \\
$C$ & 0 & 0 & 0 & 0.0122 & 0.0062 & 0.0010 & 0.0039 \\
$A C$ & 0.0259 & 0 & 0.0019 & 0.0066 & 0.0092 & 0.0006 & 0.0061 \\
$B C$ & 0 & 0.0012 & 0.0011 & 0.0033 & 0.0019 & 0.0008 & 0.0032 \\
$A B C$ & 0.0176 & 0.0015 & 0.0032 & 0.0046 & 0.0068 & 0.0010 & 0.0090 \\
\hline
\end{tabular}

TABLE 12: Transition probability matrix.

\begin{tabular}{lccccccc}
\hline & $A$ & $B$ & $C$ & $A B$ & $A C$ & $B C$ & $A B C$ \\
\hline$A$ & 0.2862 & 0 & 0.0159 & 0 & 0.0330 & 0 & 0.0325 \\
$B$ & 0 & 0.0042 & 0.0030 & 0 & 0 & 0.0120 & 0.042 \\
$A B$ & 0.0312 & 0.0012 & 0.0061 & 0 & 0.0042 & 0.0004 & 0.0612 \\
$C$ & 0 & 0 & 0 & 0.0112 & 0.0061 & 0.0011 & 0.0021 \\
$A C$ & 0.0252 & 0 & 0.0019 & 0.0063 & 0.0091 & 0.0020 & 0.0034 \\
$B C$ & 0 & 0.0011 & 0.0014 & 0.0039 & 0.0023 & 0.0012 & 0.0062 \\
$A B C$ & 0.0162 & 0.0016 & 0.0031 & 0.0041 & 0.0034 & 0.0009 & 0.0083 \\
\hline
\end{tabular}

then calculate the transferred matrix. Then, use the Dempster combination rule to calculate the final calculation result:

$m_{N+1}=\left[\begin{array}{lllllll}0.9970 & 0.0002 & 0.0000 & 0.0016 & 0.0002 & 0.0000 & 0.0000\end{array}\right]$.

Finally, the combined result at the time $N+1$ is stored and used to judge whether the evidence at the next time is conflict evidence.

Through the analysis of the transfer probability matrix, it can be seen that the transfer probability matrix has not changed greatly, which verifies the previous hypothesis; that is, the process of the source receiving evidence is an approximate Markov process, and the evidence received by the source is related to the current time, and not to the historical data.

The results show that the combination of conflict evidence with the transition probability matrix can get a good combination effect.

\section{Conclusions}

This paper realizes the problem of conflicting evidence combination in time series based on the evidence transition probability matrix. Temporal conflict evidence combination is a process that uses the evidence transition probability matrix of the previous $n$ moments, combines the current moment and the evidence of the next moment to calculate the transition matrix of the next moment, and then uses the transition matrix of the next moment to combine the evidence. This paper also combines two types of evidence transition probability matrix to propose a conflict evidence combination model. First, the model determines whether the evidence conflicts; if there is a conflict, the evidence is modified, and then the modified evidence is used to calculate the combination result. The combination results show that the evidence combination model based on the transition probability matrix can solve the combination problem of conflicting evidence well.

\section{Data Availability}

The data used to support the findings of this study are available from the corresponding author upon request.

\section{Conflicts of Interest}

The authors declare that they have no conflicts of interest.

\section{Acknowledgments}

This work was partially supported by the special fund of Taishan Scholar Project (201712072).

\section{References}

[1] L. A. Zadeh, "A simple view of the Dempster-Shafer theory of evidence and its implication for the rule of combination," $A I$ Magazine, vol. 7, no. 2, pp. 85-90, 1986.

[2] D. Dubois and H. Prade, "Representation and combination of uncertainty with belief functions and possibility measures," Computational Intelligence, vol. 4, no. 3, pp. 244-264, 1988.

[3] P. Smets, "Data fusion in the transferable belief model," in Proceedings of the Third International Conference on Information Fusion, vol. 1, Paris, France, July 2000.

[4] F. Smarandache and D. Jean, "Modified pcr rules of combination with degrees of intersections," in Proceedings of the International Conference on Information Fusion, pp. 21002107, Washington, DC, USA, July 2015.

[5] W. Jiang, "A correlation coefficient for belief functions," International Journal of Approximate Reasoning, vol. 103, pp. 94-106, 2018.

[6] C. K. Murphy, "Combining belief functions when evidence conflicts," Decision Support Systems, vol. 29, no. 1, pp. 1-9, 2000.

[7] J. Dezert and F. Smarandache, Advances and Applications of DSmT for Information Fusion Collected Works, American Research Press, Rehoboth, CT, USA, 3th edition, 2009.

[8] A.-L. Jousselme, D. Grenier, and É. Bossé, "A new distance between two bodies of evidence," Information Fusion, vol. 2, no. 2, pp. 91-101, 2001.

[9] C. Yu, J. Yang, D. Yang, X. Ma, and H. Min, “An improved conflicting evidence combination approach based on a new supporting probability distance," Expert Systems with Applications, vol. 42, no. 12, pp. 5139-5149, 2015.

[10] D. Zhou, Q. Pan, and G. Chhipi-Shrestha, "A new weighting factor in combining belief function," PLoS One, vol. 12, no. 5, 2017.

[11] W. Jiang, M. Zhuang, X. Qin, and Y. Tang, "Conflicting evidence combination based on uncertainty measure and distance of evidence," SpringerPlus, vol. 5, no. 1, p. 1217, 2016.

[12] L. Pan and Y. Deng, "A new belief entropy to measure uncertainty of basic probability assignments based on belief function and plausibility function," Entropy, vol. 20, no. 11, p. 842, 2018.

[13] R. Jirousek and P. Prakash, "A new definition of entropy of belief functions in the Dempster-Shafer theory," International Journal of Approximate Reasoning, vol. 92, pp. 49-65, 2018. 
[14] J. Abellán, "Analyzing properties of Deng entropy in the theory of evidence," Chaos, Solitons \& Fractals, vol. 95, pp. 195-199, 2017.

[15] H. Cui, Q. Liu, J. Zhang, and B. Kang, "An improved Deng entropy and its application in pattern recognition," IEEE Access, vol. 7, pp. 18284-18292, 2019.

[16] Q. Pan, D. Zhou, Y. Tang, X. Li, and J. Huang, "A novel belief entropy for measuring uncertainty in dempster-shafer evidence theory framework based on plausibility transformation and weighted Hartley entropy," Entropy, vol. 21, no. 2, pp. 163-182, 2019.

[17] Y. Deng, "Deng entropy," Chaos, Solitons \& Fractals, vol. 91, pp. 549-553, 2016.

[18] R. R. Yager, "Interval valued entropies for Dempster-Shafer structures," Knowledge-Based Systems, vol. 161, pp. 390-397, 2018.

[19] D. Zhou, Y. Tang, and W. Jiang, "An improved belief entropy and its application in decision-making," Complexity, vol. 2017, Article ID 4359195, 15 pages, 2017.

[20] L. Hong and A. Lynch, "Recursive temporal-spatial information fusion with applications to target identification," IEEE Transactions on Aerospace and Electronic Systems, vol. 29, no. 2, pp. 435-445, 1993.

[21] C.-L. Fan, Y. Song, L. Lei, X. Wang, and S. Bai, "Evidence reasoning for temporal uncertain information based on relative reliability evaluation," Expert Systems with Applications, vol. 113, pp. 264-276, 2018.

[22] M. Dugenci, "A new distance measure for interval valued intuitionistic fuzzy sets and its application to group decision making problems with incomplete weights information," Applied Soft Computing, vol. 41, pp. 120-134, 2016.

[23] L. Fei, J. Xia, Y. Feng, and L. Liu, "A novel method to determine basic probability assignment in Dempster-Shafer theory and its application in multi-sensor information fusion," International Journal of Distributed Sensor Networks, vol. 15, no. 7, Article ID 155014771986587, 2019.

[24] D. Kong, T. Chang, J. Pan et al., "A decision variable-based combinatorial optimization approach for interval-valued intuitionistic fuzzy magdm," Information Sciences, vol. 484, pp. 197-218, 2019.

[25] W. Wu, Y. Song, and W. Zhao, "Evaluating evidence reliability on the basis of intuitionistic fuzzy sets," Information, vol. 9, no. 12, p. 298, 2018.

[26] G. Wang, J. Zhang, Y. Song, and Q. Li, “An entropy-based knowledge measure for Atanassov's intuitionistic fuzzy sets and its application to multiple attribute decision making," Entropy, vol. 20, no. 12, p. 981, 2018.

[27] L. Dymova and P. Sevastjanov, "An interpretation of intuitionistic fuzzy sets in terms of evidence theory: decision making aspect," Knowledge-Based Systems, vol. 23, no. 8, pp. 772-782, 2010.

[28] X. Li, A. Dick, C. Shen, Z. Zhang, A. van den Hengel, and H. Wang, "Visual tracking with spatio-temporal DempsterShafer information fusion," IEEE Transactions on Image Processing, vol. 22, no. 8, pp. 3028-3040, 2013.

[29] I. Huerta, G. Ferrer, F. Herrero, A. Prati, and A. Sanfeliu, "Multimodal feedback fusion of laser, image and temporal information," in Proceedings of the International Conference on Distributed Smart Cameras-ICDSC'14, p. 25, Venezia, Italy, November 2014.

[30] Y. Dan, J. Hongbing, and G. Yongchan, “A robust D-S fusion algorithm for multi-target multi-sensor with higher reliability," Information Fusion, vol. 47, pp. 32-44, 2019.
[31] T.-J. Park and J.-H. Chang, "Dempster-shafer theory for enhanced statistical model-based voice activity detection," Computer Speech \& Language, vol. 47, pp. 47-58, 2018.

[32] T. Komorowski, S. Peszat, and T. Szarek, "On ergodicity of some Markov processes," The Annals of Probability, vol. 38, no. 4, pp. 1401-1443, 2010.

[33] M. Arns, P. Buchholz, and A. Panchenko, "On the numerical analysis of inhomogeneous continuous-time Markov chains," INFORMS Journal on Computing, vol. 22, no. 3, pp. 416-432, 2010. 